\title{
Field Decay and Snapback Measurements Using a Fast Hall Probe Sensor
}

\author{
T. Pieloni, S. Sanfilippo, L. Bottura, M. Haverkamp, A. Tikhov, E. Effinger, E. Benedico, and N. Smirnov
}

\begin{abstract}
During beam injection the components of the magnetic field inside the magnets decay in time. At the re-start of the ramping, this decay is cancelled resulting in a fast change of the harmonics called "snapback". This sudden variation affects mainly the sextupole and decapole components and can induce significant changes in the machine chromaticity, thus causing particle beam loss. Standard magnetic measurements with rotating coils do not have a sufficient time resolution to properly characterize the snapback phenomenon. A system based on Hall probes has been developed from an existing prototype to measure with a moderate acquisition frequency $(3-10 \mathrm{~Hz})$ the decay and snapback of the sextupole and, for the first time, of the decapole fields. The present system also provides local measurements of these field harmonics along a wavelength of the superconducting cable twist pitch. In this paper, we describe the assembly features of this detector and of the measurement chain. The performances are demonstrated based on preliminary measurements performed on the LHC pre-series dipoles in operating conditions.
\end{abstract}

Index Terms-Decay, snapback, superconducting magnets.

\section{INTRODUCTION}

$\mathbf{F}$ OR the Large Hadron Collider (LHC), a constant magnetic field of $0.54 \mathrm{~T}$ is required to inject the particle beam. During this constant current plateau the field quality inside the superconducting magnet bore changes with time: all components of the magnetic field show a decay in time. When the current ramp starts again, all the harmonics suddenly go back to their value at injection before the decay, i.e., they 'snapback' [1]. If not compensated by the correctors, this sudden variation, mainly of the sextupole and decapole multipoles, can cause particle loss. The present understanding of the origin of this phenomenon is that BICC's (Boundary Induced Coupling Currents) induced in superconducting filaments during current ramps and trapped among the strands, circulate in various loops formed by cable sections with different time constants and over different distances. During periods of constant transport current, as for example during injection, these supercurrents produce changes in the local field that reduce the filament magnetization, thus causing the field decay. When the external field is increased, the magnetization bounces back to the original hysteresis curve, which explains the behavior during the snapback [2]. In the LHC dipoles the amplitudes of the decay and the snapback are measured using 15-m long twin rotating coils [3].

Manuscript received October 21, 2003.

T. Pieloni is with the Universita' degli Studi di Milano, Dip. Fisica, 20100 Milano, Italy (e-mail: Tatiana.Pieloni@cern.ch).

S. Sanfilippo, L. Bottura, M. Haverkamp, A. Tikhov, E. Effinger, E. Benedico, and N. Smirnov are with CERN, CH-1211 Geneva 23, Switzerland.

Digital Object Identifier 10.1109/TASC.2004.830871
The rotating coil low acquisition frequency $(0.1 \mathrm{~Hz})$ is however not fast enough to measure the snapback that occurs in some tens of seconds when the current ramp starts. Moreover rotating coils have a poor spatial resolution because field components are integrated over the coil length of $1.25 \mathrm{~m}$. For this reason a Hall-plate based sensor for fast and local measurements of the decay and snapback of the sextupole and for the first time of the decapole component was developed. The $b_{3}$ and the $b_{5}$ components are essential for LHC performance because these two harmonics give systematic errors to the beam dynamic parameters. They cause changes in the chromaticity of the first and second order.

The $b_{3}$ and $b_{5}$ detector was derived from an old prototype version which included the sextupole sensors only [4]. The new device contains six sensors placed to measure the $b_{3}$ and two for the $b_{5}$. The decapole sensors were added because during the accelerator performance this harmonic will not be measured by the Beam Position Monitors (BPM). This $b_{3}-b_{5}$ sensor allows measurements of the decay and snapback at a higher acquisition frequency $(3-10 \mathrm{~Hz})$ resolving properly the phenomena. Moreover Hall plates give a point-like measurement that allows spatial resolution of the harmonics along a cable twist pitch.

This paper will describe the basic principle of the multipole measurement with the Hall plate sensor as well as the mechanics and the electronics of the device. The calibration of the detector and preliminary results of the decay and snapback of the sextupole and decapole components will be presented. It was found out during measurements on different LHC dipoles that there is an excellent correlation between the change in the sextupole decay amplitude and the current necessary to completely resolve the snapback.

\section{THE SENSOR}

In the LHC dipole magnets the 2-dimensional magnetic field is given by the multipolar expansion:

$B(x, y)=B_{y}+i B_{x}=10^{-4} B_{1} \sum_{n=1}^{\infty}\left(b_{n}+i a_{n}\right)\left(\frac{x+i y}{R_{r e f}}\right)^{n-1}$

where $b_{n}$ and $a_{n}$ are the normal and skew multipole coefficients, respectively, and $R_{r e f}=17 \mathrm{~mm}$ is the reference radius. Multipoles are expressed in units of $10^{-4}$ with respect to the main dipolar component $B_{1}$.

As explained in detail in [5], [6], it is possible to measure the $n$-th order harmonic of the dipole field using an appropriate arrangement of $n$ Hall plates. The $n$ Hall probes are placed tangentially to a ring at a radial distance $R$ and at the azimuthal 


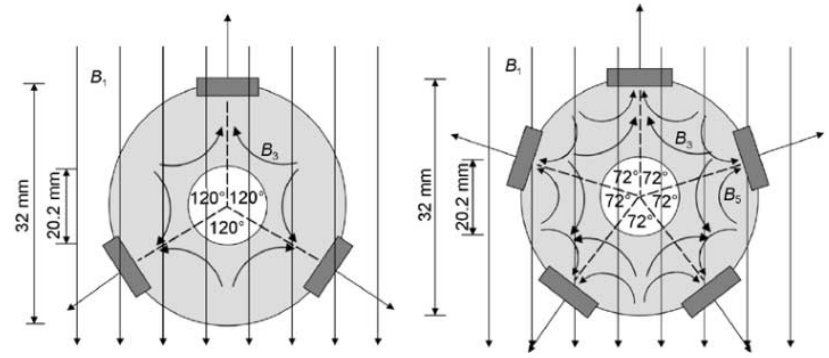

Fig. 1. Examples are shown for a sextupole ring with 3, and a decapole ring with 5 Hall plates. Also the field lines of the dipole, the sextupole and the decapole field are indicated.

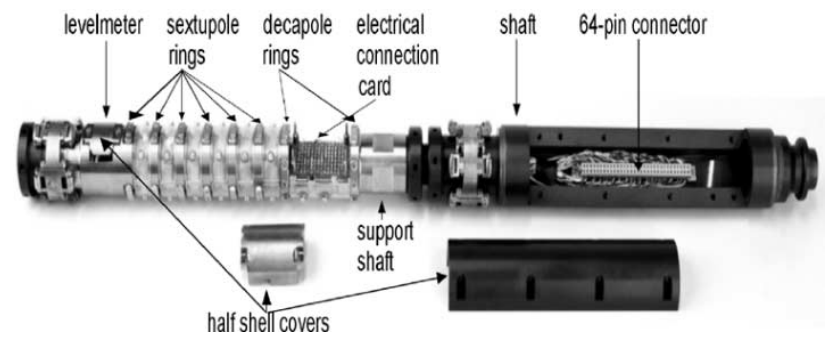

Fig. 2. A picture of the Hall plate detector.

angles $2 \pi / n$. To measure the component of order $n=3$ and $n=5$ of the field the sensor rings are arranged as shown in Fig. 1.

With this ideal geometry the total signal, $S$, from $n$ Hall plates as described above is given by:

$$
\begin{aligned}
S_{n}=\sum_{k=1}^{\infty}\left(\frac{R}{R_{\text {ref }}}\right)^{(2 k-1) n-1}(-1)^{\frac{(2 k-1) n-1}{2}} n B_{(2 k-1) n} \\
+\sum_{k=1}^{\infty}\left(\frac{R}{R_{\text {ref }}}\right)^{2 k n}(-1)^{k n} n A_{2 k n},
\end{aligned}
$$

where $k$ is an arbitrary integer. Note that in the sum only odd normal multipoles and even skew multipoles enter. The formula can be simplified recalling that, in a dipole, odd normal harmonics are allowed by symmetry. Besides they strongly decrease in amplitude with increasing order. On the other hand the even skew multipoles are not allowed by the symmetry. Thus, for a magnet with the proper geometry, they are expected to be close to zero. Hence at first order, for $n=3$ and $n=5$, (2) can be simplified as follows:

$$
S_{3} \approx 3\left(\frac{R}{R_{\text {ref }}}\right)^{2} B_{3} ; S_{5} \approx 5\left(\frac{R}{R_{\text {ref }}}\right)^{4} B_{5} .
$$

In first approximation, the signals coming from the arrangement of the sensors in the rings (Fig. 1) are proportional to the normal sextupole and decapole harmonic, respectively. The dipole field component is completely compensated by the symmetry. Note that in the case of the decapole geometry also the sextupole harmonic is compensated by the Hall probe arrangement.

The device consists of six rings for the sextupole measurements and two rings for the decapole, as shown in Fig. 2, fixed on a common shaft made of Ti6Al4V alloy to avoid mechanical misalignments of the rings. The piece is $300 \mathrm{~mm}$ long, has a diameter of $33 \mathrm{~mm}$, includes the eight rings and contains a flat surface for an electrical connection card. A hole of $15 \mathrm{~mm}$ diameter is carved inside the support shaft in order to minimize the quantity of Ti6Al4V. The six $b_{3}$ sensors are placed at a distance of $19.2 \mathrm{~mm}$, one from each other, in order to cover a wavelength of the cable twist pitch. The two $b_{5}$ rings are spaced by half a pattern wavelength $(57.5 \mathrm{~mm})$. Hall plates are mounted as shown in Fig. 1 into grooves on the rings at angular spacing of $120^{\circ}$ and $72^{\circ}$, respectively, with an angular tolerance of $\pm 1^{\circ}$.

Signals from the 28 Hall plates are connected to a distribution box that contains compensation cards: six for the sextupole and two for the decapole. These electronic cards make an analog sum of the Hall plate signals of each sensor resulting in a first compensation of the dipole component. The compensated signals are amplified by Programmable Gain Amplifiers (PGA) and read out simultaneously by a set of Precision Digital Integrators (PDI) in continuous mode; the VME-PDI's are triggered externally, by a function generator. The typical noise level in operation conditions (dipole powered at $760 \mathrm{~A}$ and the sensor with $50 \mathrm{~mA}$ ) is in the $10 \mu \mathrm{V}$ range. The integration of the signal over small time intervals allows a reduction of the noise level [7].

\section{SENSOR CALIBRATION}

Theoretically, the dipole field is erased by the sensor geometry; experimentally, an un-bucked component due to different error sources is always present. Spurious signals coming from different errors are to be taken into account: amplifiers and Hall probe temperature-dependent offsets, Hall plate angular misalignments and Hall plate nonlinear sensitivity are the most important.

In the LHC dipoles the sextupole and decapole components are in the range of a few units and a few tenths of units, respectively. To achieve an accuracy of 0.01 units for the $b_{3}$ and $b_{5}$ harmonics one would need a calibration at the $10^{-6}$ level. A reference magnet with a good homogeneity and a known dipole field in the $\mu \mathrm{T}$ range has to be used. This way of calibration was not practical because of the strong dependence of the sensors on the temperature and on the accuracy of the sensor of the longitudinal position. Therefore the absolute calibration of the sensor is carried out in two steps. First an electronic calibration is executed in order to compensate the dipole signal and to qualitatively reconstruct the hysteresis curve. The adjustments are carried out inside a reference dipole magnet permanently checked by a Nuclear Magnetic Resonance magnetometer with an accuracy of $10^{-7} \mathrm{~T}$. All the electronic offsets are adjusted at a zero level inside a dipole field of $0.537 \mathrm{~T}$ and, in order to compensate the differences in the Hall plate sensitivity and angular alignment, every pre-amplifier gain is adjusted according to the NMR value and Hall plate sensitivity. The second step is to eliminate the spurious contributions by off-line analysis, using as a reference results coming from the rotating coils. Hall plate data are reduced using the following conversion formula:

$$
b_{n}=\frac{K_{n}\left(V_{n}-V_{\text {offset }}-K_{\text {bucking }} B_{1}-K_{\text {nonlinear }} B_{1}^{2}\right)}{B_{1}} 10^{4},
$$

where $b_{\mathrm{n}}$ is the normalized sextupole for $n=3$ or decapole for $n=5, V_{n}$ is the average voltage signal from the ring sensors, $B_{1}$ is the dipole field, $V_{\text {of fset }}$ is the electronic offset from the amplifiers, $K_{n}$ is the calibration factor for the voltage of the nth plate 


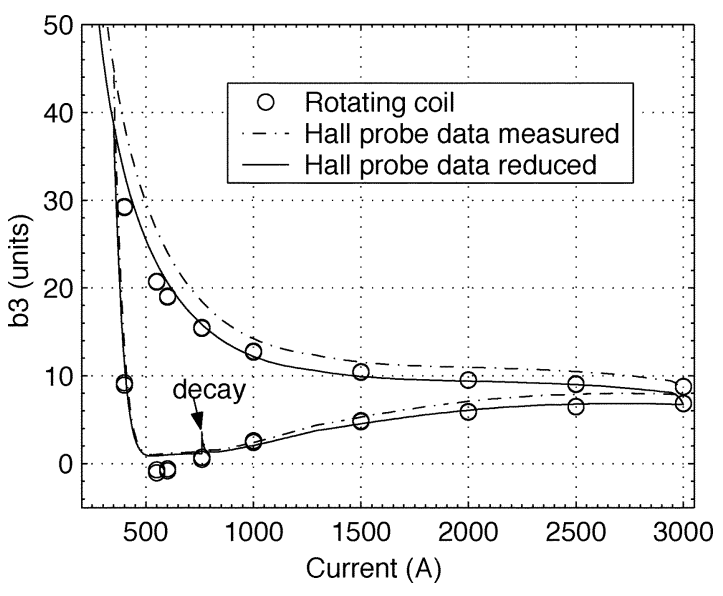

Fig. 3. Sextupole component hysteresis curve measured with rotating coils and with the Hall plate sensor, before and after the off-line calibration.

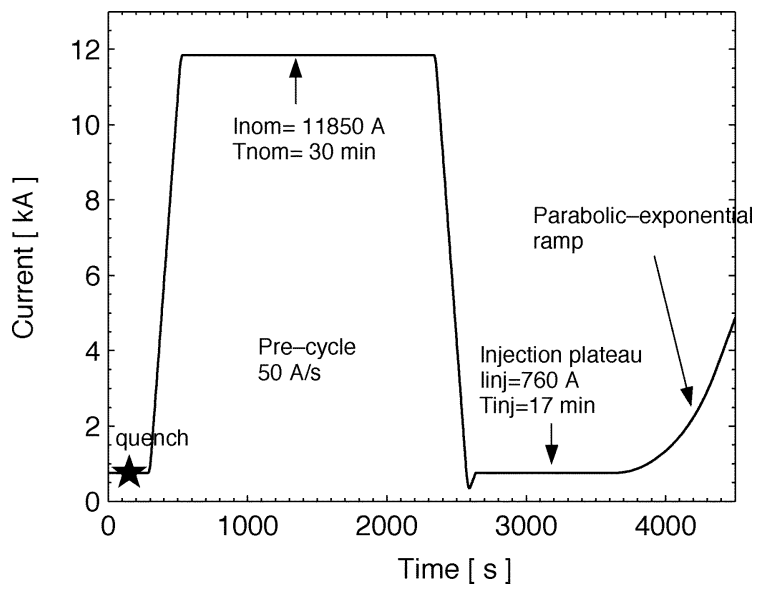

Fig. 4. The current cycle for the decay and snapback measurements in the LHC dipoles. The decay occurs during the plateau at $760 \mathrm{~A}$ and the snapback as soon as the ramp restarts.

sensor read-out, $K_{\text {bucking }}$ is the dipole voltage bucking ratio and $K_{\text {nonlinear }}$ is a second order correction for the Hall probes non linear sensitivity as a function of field. The parameters $V_{\text {off set }}$, $K_{\text {bucking }}, K_{\text {nonlinear }}$ and $K_{n}$ are obtained by an unconstrained optimization procedure that aims at minimizing the root mean square of the difference among Hall-plates reading and rotating coil results.

The result of this calibration is shown for the dipole 1009 in Fig. 3, where the Hall probe sextupole hysteresis curve, before and after the data reduction, is compared with that measured with rotating coils.

\section{MEASUREMENTS AND RESUlTS}

First measurements were performed on four different pre-series dipole magnets: 1009, 1024, 2020 and 3005.

In all cases, snapback measurements were performed using one LHC operation cycle as reference (Fig. 4). The current cycle starts with a quench and a pre-cycle in order to bring the magnet on a well known hysteresis curve. Then the current is ramped up from $350 \mathrm{~A}$ to $760 \mathrm{~A}$ at $10 \mathrm{~A} / \mathrm{s}$ and kept constant for 1000 $\mathrm{s}$ to simulate the beam injection plateau. The current is then increased up to 11850 A with a parabolic-exponential acceleration at the start of the ramp.

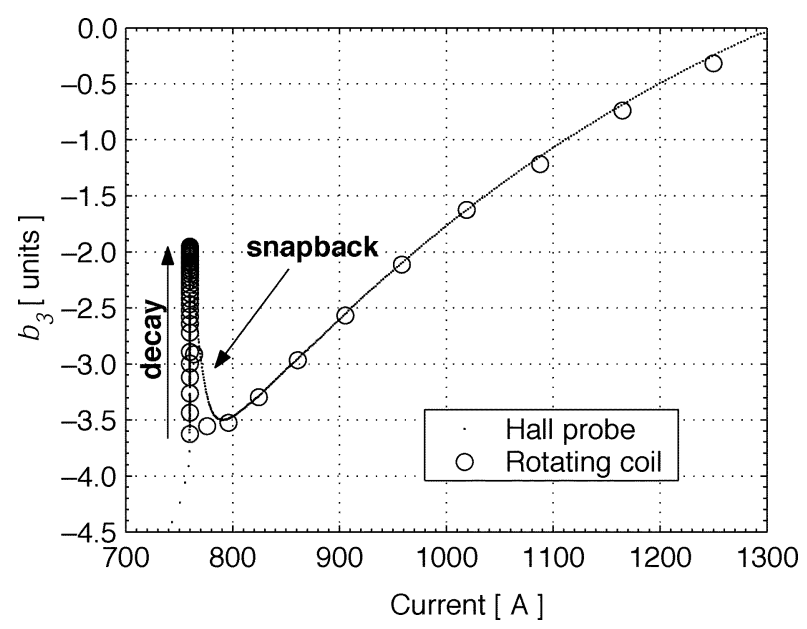

Fig. 5. The sextupole decay and snapback are shown as a function of the excitation current. The plot compares results from rotating coils and from the Hall plate sensor.

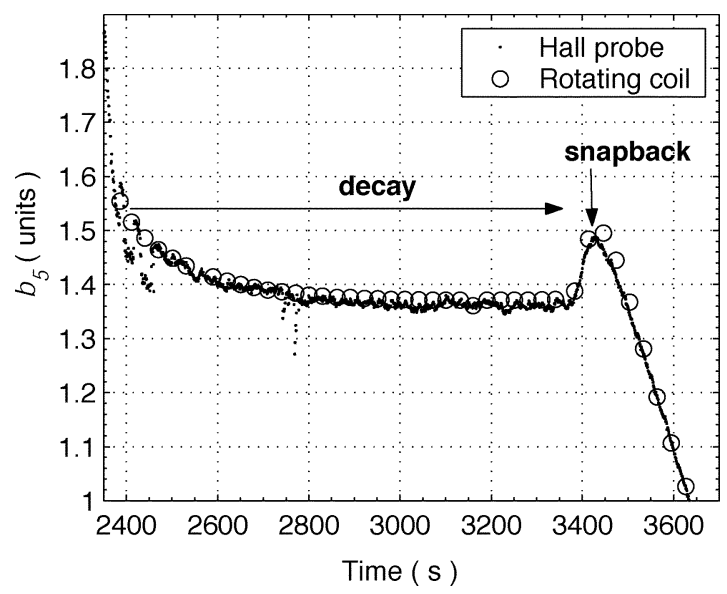

Fig. 6. The decapole decay and snapback are shown as a function of time. Hall plate data are compared with rotating coil results. A parasitic signal is visible at the start of the decay and could be due to distortion coming from the magnet power supply.

\section{A. Comparison Between Hall Plate Results and Rotating Coil Measurements}

The $b_{3}-b_{5}$ sensor was entered inside the bore at the center of the magnet. To ensure a good alignment of the probes, the sensor must be first oriented at $0.537 \mathrm{~T}$ to have the dipole field perpendicular to the top Hall plates. After the orientation, the reference cycle is performed.

Fig. 5 shows the sextupole decay and snapback measured by two rings compared to those obtained from the rotating coils. Note the difference of the number of experimental points taken during the snapback between the two systems of measurements. The two curves show a very good agreement both in shape and in amplitude (decay of 1.77 instead of 1.71 units). The differences between the two measurements during the snapback phase are in the order of 0.02 units. In the snapback phase the lower current values obtained from the rotating coil are not fully understood yet. They could be due to an under-estimation of the current value measured over a turn.

Fig. 6 shows the decapole decay and the snapback as a function of time during the injection plateau; the measurement was 


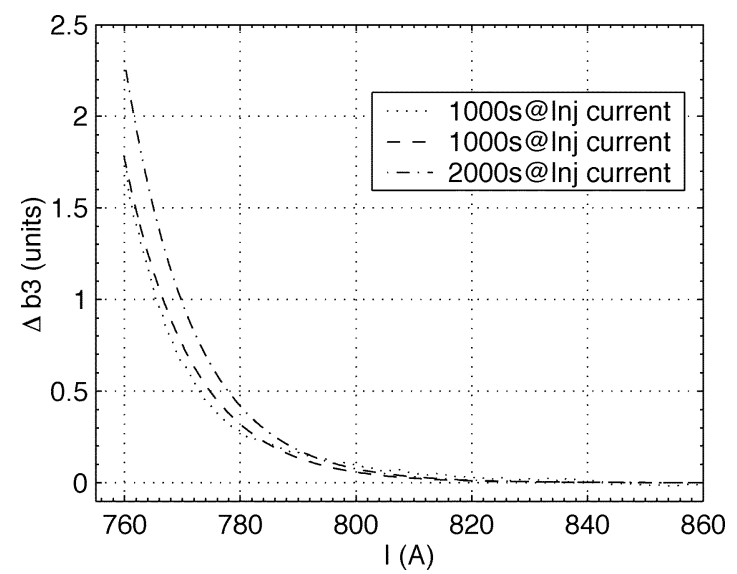

Fig. 7. Sextupole absolute change during the snap-back phase is plotted as a function of the current change. The sextupole change appears to be not correlated to the injection duration. Even if the decay amplitude is bigger for the longer plateau, the current change to reach the magnetization value of $b_{3}$ is always around $40 \mathrm{~A}$.

performed on the dipole 1024 with the same current cycle. The decapole measurements were really difficult to execute because of the small amplitude of the harmonic (one order smaller than the sextupole) and of the amplified noise due to misalignment of probes ( 5 probes instead of three). Nevertheless the decay amplitude and the snap-back shape are in accordance with the rotating coil results.

\section{B. Correlation}

To predict the harmonics behavior during the snapback we aim to find a correlation between the change in amplitude of the absolute sextupole and the change in current during the snapback phase. This correlation was already observed during a measurement campaign on the Tevatron magnets using a sextupole Hall probe sensor [8]. Measurements were performed for different magnets changing the duration of the injection current plateau. The evolution of the $b_{3}$ as a function of the current change during the snapback was studied.

To obtain the effective harmonic change due to the snapback only, the measured values are subtracted from the magnetization values of $b_{3}$ extrapolated at that current if the decay and snapback did not occur. The result of this analysis is shown in Fig. 7, where the change of the sextupole during the snapback as a function of current for different current cycles is presented. From direct and numerical experience [8], we use an exponential curve to fit the absolute sextupole change obtained as a function of the current:

$$
b_{3}^{\text {snap-back }}(t)=\Delta b_{3} e^{-\frac{I(t)-I_{\text {injection }}}{\Delta I}} .
$$

The two parameters $\Delta b_{3}$ and $\Delta I$ are found to be linearly correlated for the four LHC dipoles measured (Fig. 8). This finding will be useful for the operation of the LHC. The BPM will measure the change of chromaticity during the beam injection i.e., the change in the sextupole harmonic. From the knowledge of $\Delta b_{3}, \Delta I$ will be known for a given magnet and so it will be possible to extrapolate the change of the sextupole during the snapback in order to help the corrector system.

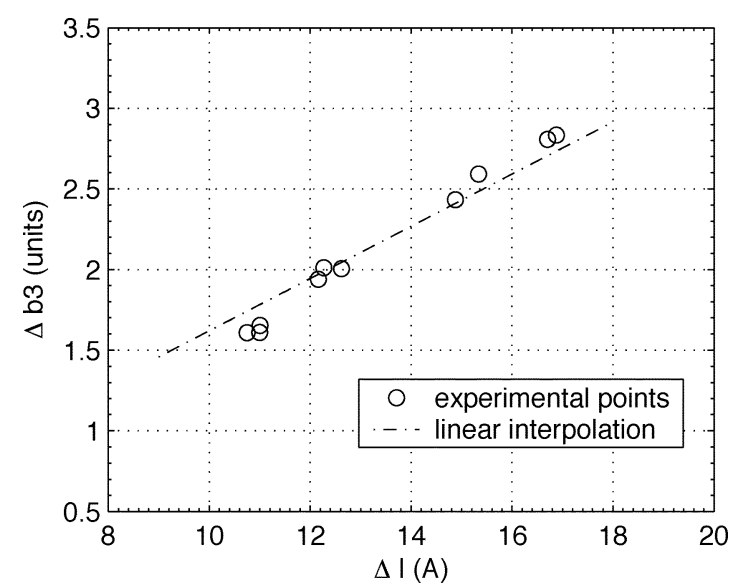

Fig. 8. Scatter plot of the snapback amplitude change $\Delta b_{3}$ versus the current change $\Delta I$ for all cycles and magnets tested.

\section{CONCLUSIONS}

A $b_{3}$ and $b_{5}$ Hall probe sensor was used to measure the decay and the snapback of the sextupole and decapole components in several LHC dipoles. Preliminary measurements compared to those obtained from rotating coil are presented. The device gives excellent results and opens the possibility to better understand the physics of the decay and snapback phenomena. For the sextupole measurements a correlation was found between the harmonic change during the snap-back and the current variation inside the magnet. We aim to confirm this correlation with measurements on more magnets; if confirmed it could be used for the online-compensation of decay and snapback in the LHC machine as the harmonics value during the snapback could be evaluated as a function of the magnet current with a simple interpolation.

\section{ACKNOWLEDGMENT}

The authors wish to thank the AT-MTM-OP team that helps in the execution of the magnetic measurements, the AT-MTM-TF section for providing and maintaining the equipment and the AB-CO-LS section for producing the acquisition software.

\section{REFERENCES}

[1] L. Bottura, L. Walckiers, and R. Wolf, "Field decay and snap-back in LHC model dipoles," IEEE Tran. Appl. Supercond., vol. 7, no. 2, pp. 602-605, June 1997.

[2] R. Wolf, "The decay of the field integral in superconducting accelerator magnets wound with rutherford cables," in Proc. of 15th Int. Mag. Tech. Conf.. Beijing, 1997, pp. 238-241.

[3] J. Billan et al., "Twin rotating coils for cold magnetic measurements of $15 \mathrm{~m}$ long LHC dipoles," IEEE Trans. Appl. Supercond., vol. 10, no. 1, pp. 1422-1426, March 2000.

[4] M. Haverkamp et al., "Field decay and snapback measurements using a fast hall plate detector," IEEE Trans. Appl. Supercond., vol. 12, no. 1, pp. 86-89, March 2002.

[5] M. Breschi et al., "Fast Measurement of Field Harmonics Through a Set of All Probes," unpublished, 2000.

[6] L. Bottura et al., "A fast sextupole probe for snapback measurements in the LHC dipoles," IEEE Trans. Appl. Supercond., vol. 10, no. 1, pp. 1435-1438, March 2000.

[7] M. Haverkamp, "Decay and Snapback in Superconducting Accelerator Magnets," Ph.D. thesis, Twente University Press, 2003.

[8] L. Bottura, P. Bauer, and G. Velev, "Main Results of the Sextupole Measurements on Tevatron Dipole," unpublished, April 2003. 